ARTICLE

\title{
Photon Energy Spectrum Reconstruction Based on Monte Carlo and Measured Percentage Depth Dose in Accurate Radiotherapy
}

\author{
Gui $\mathrm{LI}^{1,2,3,}$, Huaqing ZHENG ${ }^{1,3}$, Guangyao $\mathrm{SUN}^{2,3}$ and Yican $\mathrm{WU}^{1,2,3}$ \\ ${ }^{1}$ Institute of Plasma Physics, Chinese Academy of Sciences, \\ Hefei, Anhui, 230031, China \\ ${ }^{2}$ School of Nuclear Science and Technology, University of Science and Technology of China, \\ Hefei, Anhui, 230027, China \\ ${ }^{3}$ Engineering Technology Research Center of Accurate Radiotherapy, AnHui Province, Hefei, Anhui, 230031, China
}

\begin{abstract}
In accurate radiotherapy, energy spectra of high energy x-ray greatly affect the accuracy of dose calculation. Conventional discrete method failed to realize the stable solution, and the Schiff formula could not reflect the practical geometric shape of different linac system that showed the limitation to apply to other linacs. To obtain the photon energy spectra of linac in radiotherapy effectively, by use of monoenergetic percentage depth dose (PDD) simulated by Monte Carlo and measured PDD, an improved analytical model based on improvement of the Schiff formula was investigated, and several regression algorithms including Levenberg-Marquardt, Quasi-Newton, Gradient, Conjugate-Gradient, Newton, Principal-Axis and Nminimize algorithms were used to realize this model. The results were also compared with the conventional discrete method.

A representative testing sample of Mohan6 (Monte Carlo simulated sample) and two representative samples of application (AAPM-55\# report at an 18 MV's AECL Therac-20 medical linac, and our measurement at a 6 MV's BJ6-B medical linac) were used to test our method. The testing sample showed its accurate and stable results of the improved analytical method that the mean error could arrived at $0.2 \%$ to the reconstructed photon energy spectra, and the mean error of the reconstructed PDD could be at $0.003 \%$ in analytical method. By comparison with the discrete method, we found that even though the mean error in reconstructed PDD of the discrete method reduced to $0.002 \%$, the discrete one with all above algorithms showed worse and unstable solution of photon energy spectra. And in clinical application samples, the analytical method also showed the same conclusion. So the conventional discrete method in reconstruction of energy spectra could be unreliable even though its reconstruction PDD agreed well to the original one. The improved analytical method also showed that it overcame the limitation of the Schiff formula that ignored various geometric shape of entire filtering system. So the improved analytical method to unfold photon energy spectra could be expected to be applied to various practical linac systems to obtain more accurate photon energy spectra.
\end{abstract}

KEYWORDS: energy spectrum, Monte Carlo, radiotherapy, dose calculation, linac

\section{Introduction}

The photon energy spectra are the important parameters in both radiotherapy and accelerator physics. Regardless of the Monte Carlo simulation or analytic method used for dose calculations, photon energy spectra play a critical role in improving dose accuracy. Presently, there are mainly three ways to obtain the photon energy spectra: modeling source by the Monte Carlo simulation, ${ }^{1)}$ measurement by Compton Scatter Spectrometer etc, ${ }^{2,3)}$ and reconstruction by a mathematical model using the percentage depth dose (PDD) or other clinical measured data. The last way seems an effective way, because it only needs to construct the suitable mathematical model.

Deng et al. reported that a random creep algorithm based on Monte Carlo simulations can reconstruct the photon energy spectra for Varian medical linac. ${ }^{4}$ Benjamin Armbruster et al. developed a linear method to reconstruct

*Corresponding author, E-mail: gli@ipp.ac.cn the photon energy spectra; ${ }^{5)}$ though their results have low accuracy, their method can obtain the continuous photon energy spectra. The low accuracy may be a result of the PDD curves lowly responding to the photon energy spectra, and the linearization may lead to larger errors.

In conventional methods, the linearization method for photon energy spectra was a kind of discrete method, in which the photon energy spectra was not treated as a mathematical analytical formula. Yet, the discrete method may lead to larger errors because in principle it is not accurate enough for the nonlinear equations. Hence, these previous studies like above are confined by the limitations of the nonlinear mathematical equation that are associated with the ill-conditioned state of this reconstruction problem, a Fredholm equation of the first kind relating the PDD data to the photon energy spectra. Furthermore, a single algorithm relative to this mathematical model may fail to solve some samples reported, so more algorithms should be applied to find the optimum solution.

Wassim $\mathrm{T}$ Jalbout et al. recently reported a analytical me- 
thod by scatter analysis to unfold photon energy spectra with the Schiff formula. ${ }^{6)}$ However, their method was based on a simplified linac model that ignored the geometric shape that showed all types of the photon energy spectra were the same regardless of the different geometric of target, flattening filter etc in different linacs. Furthermore, their method also required further to improve on the accuracy of the background and scatter measurements and the stability of the results.

The FDS Team has been working to develop series of Advanced/Accurate Radiotherapy Systems (ARTS) and the Monte Carlo Automatic Modeling Program for Particle Transport Simulation (MCAM) ${ }^{7-10)}$ etc. In the present study, based on these systems, one of the technologies, the photon energy spectrum reconstruction is realized by constructing the nonlinear mathematical model with an improved analytical method, and it is compared with the conventional discrete method. Several algorithms are used to realize the solution of this model. This work will support a series of our studies on dose calculation and human modeling, ${ }^{11-15)}$ positioning, ${ }^{16,17)}$ etc.

\section{Methodology}

\section{Conventional Discrete Method}

If it is supposed that a photon beam with energy spectrum $\Phi(E)$ normally enters into water, its PDD in center axis can be expressed as the convolution between monoenergetic depth dose $D(E, z)$ and $\Phi(E)$. Namely,

$$
D(z)=\int D(E, z) \Phi(E) d E .
$$

In the above equation, it is assumed that the dose distribution of electron contamination could be ignored. This assumption means the photon beam can be controlled better from the head of the medical linac.

In conventional discrete methods, the energy deposition of the photon beam with the energy spectrum is regarded as the linear superimposition of many monoenergetic photon beams. Supposing the photon energy spectrum equation read as a discrete formula $\Phi\left(E_{i}\right)=a_{i}$ and considering to increase the precise of the discrete method, Eq. (1) can be written as the trapezoidal sum:

$$
D(z)=\sum_{i=1}^{N-1}\left[\frac{a_{i} D\left(E_{i}, z\right)+a_{i+1} D\left(E_{i+1}, z\right)}{2}\right] \Delta E .
$$

Comparing with Eqs. (1) and (2), the coefficient $a_{i}$ is the discrete photon energy spectra $\Phi\left(E_{i}\right)=a_{i}$ with normalization. It is therefore the Eq. (2) becomes a nonlinear equation with $N$ freedoms.

The mean error is used to assess the accuracy of the reconstructed PDD $D^{\prime}(z)$ :

$$
\sigma=\sqrt{\sum_{j}^{m}\left[D^{\prime}\left(z_{j}\right)-D\left(z_{j}\right)\right]^{2}} / m,
$$

where $m$ is the number of measured PDD data used to calculate, according to the definition of mean error. Based on the above discussion, the issue of photon energy spectrum re- construction can be attributed to solving a nonlinear programming problem; the object is the minimum mean error, subjected to the nonnegative energy spectra. When introduced the absolute sign in Eq. (3), this model becomes an unconfined optimization problem:

\section{Min $\sigma$.}

The above method was the conventional discrete method, in which the photon energy spectrum equation reads as a discrete formula $\Phi\left(E_{i}\right)=a_{i}$. In this discrete model, the photon energy spectrum can be obtained by using the nonlinear regression algorithms, when measured PDD $D(z)$ and monoenergetic depth dose $D(E, z)$ are known.

However, the conventional discrete model has its inaccuracy because the Eq. (1) is an ill-state equation, a Fredholm equation. So in general, when Eq. (1) was replaced by the inaccurate discrete photon energy spectra as $\Phi\left(E_{i}\right)=a_{i}$, a very small error of measured $D(\mathrm{z})$ will lead to a large error of $\Phi(E)$. What is a better way to improve it may be use of a more accurate model, that is, an analytical formula of photon energy spectra. So in the next section we will develop an analytical method by modifying the Schiff formula to improve the accuracy of the photon energy spectrum reconstruction.

\section{Improved Analytical Method}

Schiff developed an analytical formula of photon energy spectra to a simplified linac model, ${ }^{8,18)}$

$$
\left\{\begin{array}{l}
\Phi(E)=\frac{e^{-\mu(E) t}}{E}\left[\frac{T-E}{T}(\ln \eta-1)+\left(\frac{E}{T}\right)^{2}(\ln \eta-0.5)\right] \\
\eta=\left[\left(\frac{0.511 E}{2 T(T-E)}\right)^{2}+\left(\frac{Z^{1 / 3}}{111}\right)^{2}\right]^{-1 / 2}
\end{array}\right.
$$

where $T$ is the total energy of electron incident on the target, $t$ is the hypothetical thickness of target material equivalent in attenuation to the entire filtering system (target, flattening filter etc) and $\mu(E)$ is the total linear attenuation coefficient for the target's material, and $Z$ is the effective atomic number for all attenuating materials.

However, we found that Eq. (5) ignored the various geometric shape of the entire filtering system, so it cannot apply to different commercial linac system. In the following discussion, we want to make an improvement of this formula.

It is supposed that various geometric shape of the entire filtering system of different linac will lead to change the peak and width of the photon energy spectra. In order to reflect this change, we introduce a log-normal function,

$$
f(E)=c e^{-\ln ^{2}\left(E / E_{p}\right) / 4 a^{2}}
$$

Here, $E_{p}$ is the peak value, $a$ is the half width of the peak, and $c$ is the normalization coefficient. Such function was used by Jun Deng to initialize his model to unfold the photon contamination of electron beam. ${ }^{4}$

We then introduce Eq. (6) to Eq. (5) by $\Phi(\mathrm{E}) \times f(\mathrm{E})$, and obtain the improved Schiff formula, 


$$
\left\{\begin{array}{l}
\Phi(E)=\frac{c e^{-\mu(E) t-\ln ^{2}\left(E / E_{p}\right) / 4 a^{2}}}{E} \times \\
{\left[\frac{T-E}{T}(\ln \eta-1)+\left(\frac{E}{T}\right)^{2}(\ln \eta-0.5)\right]} \\
\eta=\left[\left(\frac{0.511 E}{2 T(T-E)}\right)^{2}+\left(\frac{Z^{1 / 3}}{111}\right)^{2}\right]^{-1 / 2}
\end{array}\right.
$$

When Eq. (7) replaces Eq. (2), the conventional discrete method becomes the improved analytical method, and the photon energy spectra was depicted by an improvement of Schiff formula in various linac systems, which is defined by only six parameters as $T, Z, t, a, c$ and $E_{p}$.

\section{Results}

\section{Calculation}

According to the above mathematical model, the monoenergetic depth dose $D(E, z)$ should be obtained firstly. The $D(E, z)$ was obtained by using the EGSnrc, $\left.{ }^{19}\right)$ with particle number $5 \times 10^{8}$, point source, $10 \mathrm{~cm} \times 10 \mathrm{~cm}$ field, $\mathrm{SSD}=100 \mathrm{~cm}$, and other default parameters for monoenergetic photon beams from $1 \mathrm{MeV}$ to $30 \mathrm{MeV}$ (energy interval $0.5 \mathrm{MeV}$ ); and the statistical error less than $0.5 \%$. The linear interpolation technique was used to obtain the continuous curves. These data only needed to be simulated once and was used as a based database.

The nonlinear programming model was solved by use of the 'NonlinearRegression' package in Mathematica6.0. Seven algorithms, Levenberg-Marquardt (LE), Quasi-Newton (QU), Gradient (GR), Conjugate Gradient (CO), Newton (NE), Principal-Axis (PR) and Nminimize (NM) were available in this package. ${ }^{20)}$

By use of above method, the energy spectrum reconstruction software was developed. And it was proved to be running correctly and effectively by a lot of testings. The following section showed a representative testing by Mohan6 photon energy spectra (Mohan-6MV) -- a Monte Carlo simulated sample, from a $6 \mathrm{MV}$ 's Varian medical linac, photon energy spectrum named as Mohan6 in the EGSnrc package. It was used to input into the Monte Carlo simulation software as EGSnrc, ${ }^{21)}$ its simulated results include PDD (as an assumed measured PDD), with statistical error less than $0.5 \%$. And two applications in practical medical linac are also shown in the following section. One was the 18 MV's AECL Therac-20 medical linac (AAPM-18MV), of which the measured data were taken from a 18 MV's medical linac of AAPM Report No. 55: document "10OF.18x"-$18 \mathrm{MV} 10 \times 10 \mathrm{~cm}^{2}$ open field. ${ }^{22)}$ The other is a $6 \mathrm{MV}$ 's BJ6-B medical linac $(B J 6-B-6 M V), 10 \times 10 \mathrm{~cm}^{2}$ open field, whose data were taken from our measurement at Engineering Technology Research Center of Accurate Radiotherapy, AnHui Province, China.

In the following testing and application, the computer operation system used was a system Win XP, Intel Core2 Duo CPU (2.40 GHz+2.39 GHz), 2.4 GRAM.

\section{Testing and Application}

Table 1 shows the mean errors $\sigma$ in the discrete and the improved analytical method respectively in each sample, where the $\sigma$ is the same meaning as Eq. (3). Figures 1-3 are the reconstructed energy spectra; Fig. 1 shows the testing sample of Mohan-6MV and compares with its original energy spectrum; Fig. 2 and Fig. 3 are the application samples of AAPM-18MV and BJ6-B-6MV, respectively.

\section{Discussion}

In the testing of improved analytical method to Mohan-6MV, the mean error of reconstructed energy spectra can reach $0.2 \%$, and the mean error of reconstructed PDD at above seven regression algorithms except PR and NE algorithms $(0.013 \%)$ can reach the range between $0.003 \%$ and $0.005 \%$ in the improved analytical method (as shown in Table 1). On the other hand, though the mean error of the discrete method reduces to $0.002 \%$, its algorithms show worse and unstable solution of photon energy spectra. From Fig. 1-3, we also find that the half width and the peak of energy spectra are various due to different types of geometric shape etc of linac and the solutions are also stable. Therefore we can conclude that our method could obtain more accurate photon energy spectra, and that the method made an improvement to the Schiff formula.

Table 1 Mean error

\begin{tabular}{ccccccc}
\hline & \multicolumn{2}{c}{ Mohan-6MV } & \multicolumn{2}{c}{ AAPM-18MV } & \multicolumn{2}{c}{ BJ6-B-6MV } \\
\hline & $\begin{array}{c}\text { Analytical } \\
\sigma(\%)\end{array}$ & $\begin{array}{c}\text { Discrete } \\
\sigma(\%)\end{array}$ & $\begin{array}{c}\text { Analytical } \\
\sigma(\%)\end{array}$ & $\begin{array}{c}\text { Discrete } \\
\sigma(\%)\end{array}$ & $\begin{array}{c}\text { Analytical } \\
\sigma(\%)\end{array}$ & $\begin{array}{c}\text { Discrete } \\
\sigma(\%)\end{array}$ \\
\hline LE & 0.003 & 0.002 & 0.104 & 0.174 & 0.054 & 0.0543 \\
\hline QU & 0.005 & 0.004 & 0.104 & 0.141 & 0.054 & 0.0561 \\
\hline GR & 0.005 & 0.015 & 0.104 & 0.155 & 0.054 & 0.108 \\
\hline CO & 0.005 & - & 0.104 & - & 0.057 & - \\
\hline NE & 0.013 & - & 0.103 & - & 0.057 & - \\
\hline PR & 0.013 & - & 0.107 & - & 0.091 & - \\
\hline NM & 0.003 & - & 0.104 & - & 0.054 & 0.0514 \\
\hline
\end{tabular}

Note: the "-" means a failure of the calculation. The discrete interval of energy spectra was $0.1 \mathrm{MeV}$ for discrete 

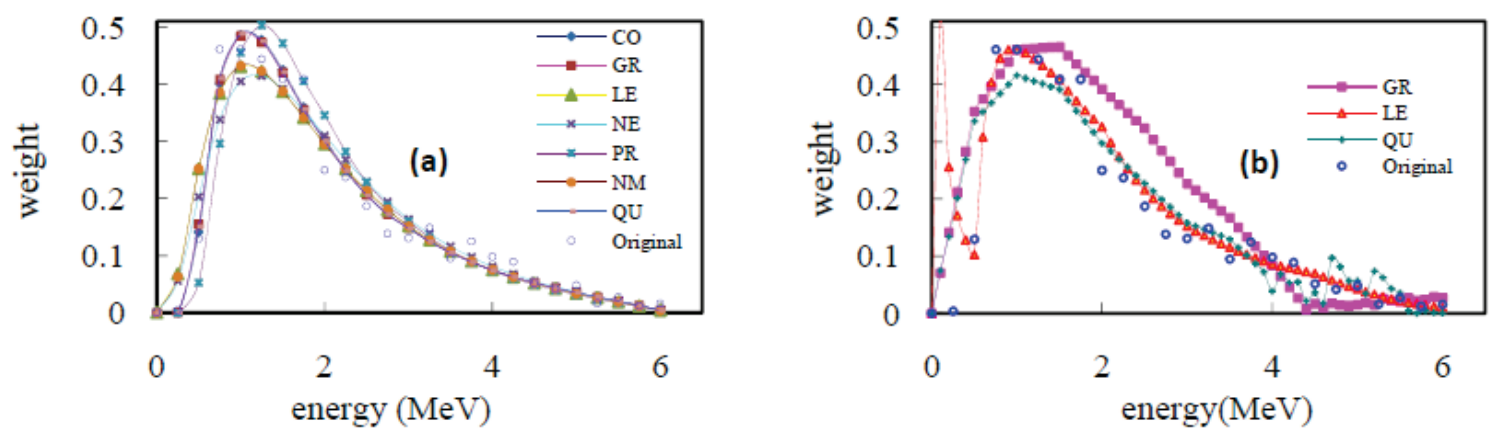

Fig. 1 Mohan-6MV energy spectra: (a) improved analytical method and (b) discrete method. The weights of energy spectra are normalized, and both results by (a) and (b) are compared with the original energy spectrum (Mohan6). Fig.1 (a) shows the close accuracy that the mean error could arrive at $0.2 \%$ (LE and NM algorithms), Mean Error $=\operatorname{Sqrt}\left(\sum \mathrm{m}(\text { Reconstructed }- \text { Original })^{2}\right) / \mathrm{m}$, while Fig. 1(b) shows the great error and unstable solution in different algorithms.
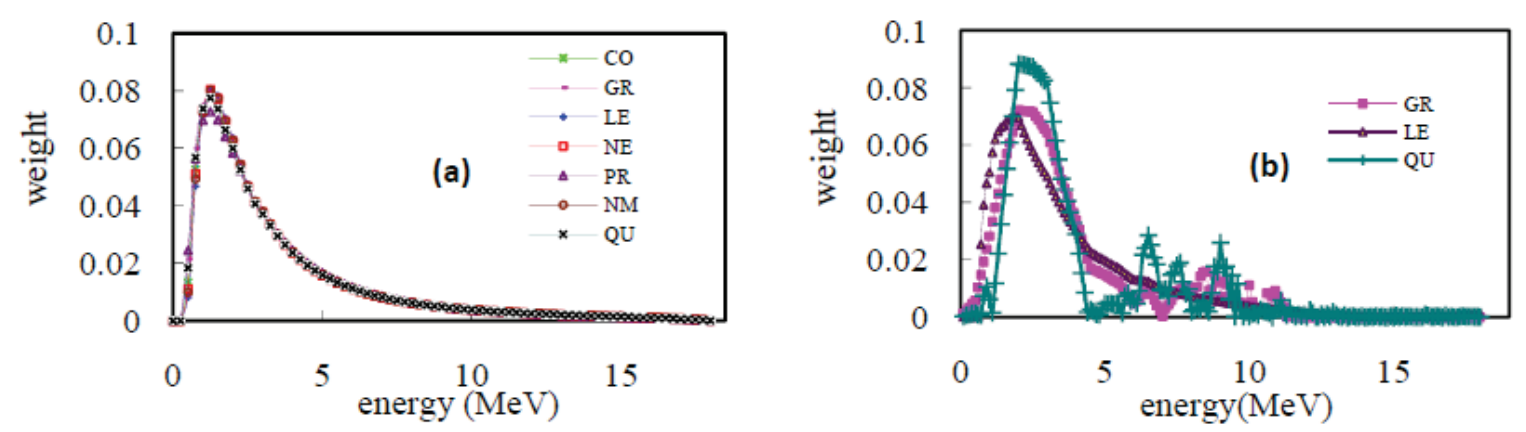

Fig. 2 AAPM-18MV energy spectra: (a) improved analytical method and (b) discrete method
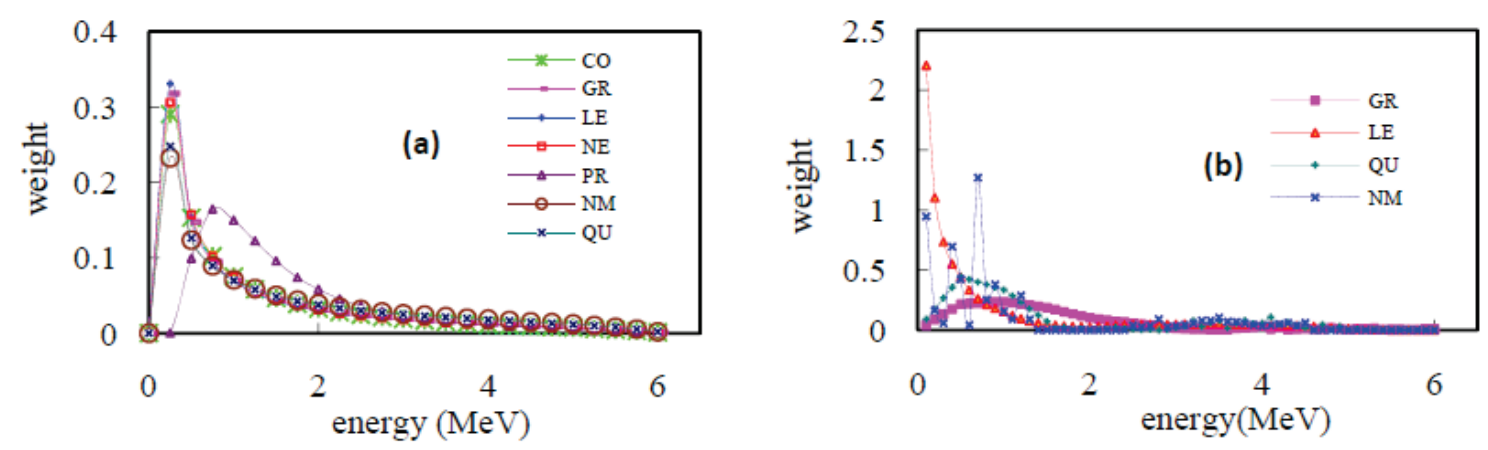

Fig. 3 BJ6-B-6MV energy spectra: (a) improved analytical method and (b) discrete method

Why the accuracy of the conventional discrete method is worse than the improved analytical one? The reason may be that Eq. (1) is an ill-conditioned state equation, a Fredholm equation, and it means that if the function of the photon energy spectra is approximated with lots of parameters, the solution usually becomes unstable and may be incorrect. And if the function of photon energy spectra is more certain with the improved analytical method using only several parameters, the solution could become more stable, just as shown in the above results.

There are also some issues we should carefully consider. For one thing, the field size also affects the photon energy spectrum reconstruction, because the photon beam impacts the boundary of the field size frequently. So we suggest it should be recalculated for different field size by using the corresponding monoenergetic PDD and the measured PDD. For another, the electron contamination was neglected in our model, and Zhu and Yang et al reported this effect was serious in some medical linacs. ${ }^{23,24)}$ In this situation, we have developed a double-source model based on the above improved analytical model, and it will be further discussed in our following paper. In addition, in the practical application, the energy fluence of the medical linac is also an important parameter that will also affect the dose calculation. So in the following study, we will also consider the energy fluence by use of additional measured data such as off-axis dose.

\section{Conclusions}

An improved analytical model by modifying the Schiff formula was proposed to reconstruct the photon energy 
spectra for medical linacs. Seven algorithms including Levenberg-Marquardt, Quasi-Newton, Gradient, Conjugate-Gradient, Newton, Principal-Axis and Nminimize algorithms were used to solve our model. They show that the reconstructed photon energy spectra agreed well with the original that the mean error of reconstructed PDD was $0.003 \%-0.013 \%$ and that the mean error of reconstructed photon energy spectra could reach $0.2 \%$ (Levenberg-Marquardt and Nminimize algorithms).

By comparison with the conventional method in unfolding the photon energy spectra in three types of representative linacs, we found that even though the mean error in reconstructed PDD of the discrete method could reduce to $0.002 \%$ and show better results than the improved analytical one, the discrete method with all above seven algorithms showed worse and unstable solution of photon energy spectra. Furthermore, in clinical application samples, the discrete method also shows the more unstable solution than the improved analytical one, even though the former mean error was better than the latter. So, we concluded that the conventional discrete method in reconstruction of photon energy spectra could be unreliable even though its reconstruction PDD agreed well with the original one.

This improved analytical method also shows that it overcomes the limitation of the Schiff formula that ignored the various geometric shape of the entire filtering system, and the method can be expected to be applied in various practical linac systems.

We will further consider the electron contamination, the field size effect and energy fluence etc in the next study on reconstruction to high energy $x$-ray of medical linac by developing a double-source model.

\section{Acknowledgment}

This work was support by the National Natural Science Foundation under grant No.30900386 and the Anhui Provincial Natural Science Foundation under grant No. 090413095. We thanked FDS Team to provide most of technology supports in this research.

\section{References}

1) G. X. Ding, "Energy spectra, angular spread, fluence profiles and dose distributions of 6 and 18 MV photon beams: results of Monte Carlo simulations for a Varian 2100EX accelerator," Phys. Med. Biol., 47[7], 1025-1046 (2002).

2) D. J. Landry, D. W. Anderson, "Measurement of Accelerator Bremsstrahlung Spectra with a High-Efficiency Ge Detector," Med. Phys., 18[3], 527-532 (1991).

3) G. Matscheko, R. Ribberfors, "A Compton-Scattering Spectrometer for Determining X-Ray Photon Energy-Spectra," Phys. Med. Biol., 32[5], 577-594 (1987).

4) J. Deng, S. B. Jiang, T. Pawlicki, J. S. Li, C. M. Ma, "Derivation of electron and photon energy spectra from electron beam central axis depth dose curves," Phys. Med. Biol., 46[5], 1429-1449 (2001).

5) B. Armbruster, R. J. Hamilton, A. K. Kuehl, "Spectrum reconstruction from dose measurements as a linear inverse problem," Phys. Med. Biol., 49[22], 5087-5099 (2004).

6) W. T. Jalbout, N. M. Spyrou, "Spectral reconstruction by scatter analysis for a linear accelerator photon beam," Phys. Med. Biol., 51[9], 2211-2224 (2006).

7) Y. Wu, L. Hu, P. Long, Y. Luo, Y. Li, Q. Zeng, L. Lu, J. Zhang, J. Zou, D. Xu, Y. Bai, T. Zhou, h. Chen, L. Peng, Y. Song, Q. Huang, "Development of design and analysis software for advanced nuclear systems," Chin. J. Nucl. Sci. Eng., 30[1], 55-64 (2010).

8) Y. WU, G. LI, S. TAO, A. WU, L. KONG, B. LIU, D. LIN, Y. CHEN, G. SONG, P. ZHAO, H. LIN, C. CHEN, Q. HUANG and L. WU, "Research and Development of an Accurate/Advanced Radiation Therapy System (ARTS)," Chin. J. Med. Phys., 22 [6], 683-690 (2005). [in Chinese]

9) $\mathrm{Y} . \mathrm{Wu}$ and $\mathrm{X} . \mathrm{Xu}$, "The Need for Further Development of CAD/MCNP Interface Codes," Trans. Am. Nucl. Soc., 96, 961-882 (2007).

10) Y. C. Wu, G. Song, R. F. Cao, A. D. Wu, M. Y. Cheng, Z. D. Tang, G. Li, J. Jing, H. Liu, J. Li, H. Y. Lan, Y. Meng, H. Q. Zheng, C. F. Jin, Q. Zeng, S. L. Zheng, Q. Y. Huang, L. J. Wu, G. L. Li, H. Lin, S. X. Tao, C. B. Shi, "Development of Accurate/Advanced Radiotherapy Treatment Planning and Quality Assurance System (ARTS)," Chin. Phys., C32, 177-182 (2008).

11) C. B. Chen, Q. Y. Huang, Y. C. Wu, "Effects of CT based voxel phantoms on dose distribution calculated with Monte Carlo method," Plasma Sci. Technol., 7[2], 2777-2780 (2005).

12) J. Jing, X. Pei, R. F. Cao, H. Lin, M. Y. Cheng, Y. C. Wu, "Optimal Grouping Algorithms for Step-and-shoot MLC Delivery in Intensity-Modulated Radiation Therapy," Int. Info. Inst., 14[3], 969-974 (2011).

13) G. Li, H. Lin, A. D. Wu, G. Song, Y. C. Wu, "Realization and comparison of several regression algorithms for electron energy spectrum reconstruction," Chin. Phys. Lett., 25[7], 2710-2713 (2008)

14) H. Lin, Y. C. Wu, Y. X. Chen, "'A finite size pencil beam for IMRT dose optimization' - a simpler analytical function for the finite size pencil beam kernel," Phys. Med. Biol., 51[6], L13-L15 (2006).

15) H. Zheng, G. Song, G. Li, " Influence of Transition Region's Thickness on Calculated Time and Precision in Region Monte Carlo Dose Calculation," Scienceonline, http://www.paper.edu.cn (2008).

16) S. Tao, Y. Wu, Y. Chen, J. Zhang, "Repeated Positioning in Accurate Radiotherapy Based on Virtual Net Technique and Contrary Reconstruction Scheme," Comput. Med. Imag. Grap., 30[5], 273-278. (2006).

17) S. Tao, A. Wu, Y. Wu, Y.Chen, J. Zhang, "Patient Set-up in Radiotherapy with Video-based Positioning System," Clin. Oncol., 18[4], 363-366 (2006).

18) G. E. Desobry, A. L. Boyer, "Bremsstrahlung Review - an Analysis of the Schiff Spectrum," Med.Phys., 18[3], 497-505 (1991).

19) EGSnrc, http://www.irs.inms.nrc.ca/EGSnrc/EGSnrc.htmt

20) Mathematica6.0, http://www.wolfram.com

21) R. Mohan, C. Chui, L. Lidofsky, "Energy and Angular-Distributions of Photons from Medical Linear Accelerators," Med. Phys., 12[5], 592-597 (1985).

22) AAPM, Report No. 55: document "10OF.18x", http://www.aapm.org/pubs/reports/report55.asp

23) J. Yang, J. S. Li, L. Qin, W. Xiong, C. M. Ma, "Modelling of electron contamination in clinical photon beams for Monte Carlo dose calculation," Phys. Med. Biol., 49[12], 2657-2673 (2004).

24) T. C. Zhu, J. R. Palta, "Electron contamination in 8 and 18 MV photon beams," Med. Phys., 25[1], 12-19 (1998). 